\title{
Flora da Reserva Ducke, Amazonas, Brasil
}

Michael J.G. Hopkins ${ }^{1}$

\section{Abstract}

(Flora of the Ducke Reserve, Central Amazon, Brazil) The Ducke Reserve in the central Amazon was the subject of an intensive floristic study between 1992 and 1999. The project showed that the biodiversity of the Reserve was much higher than had been anticipated. Data are presented chronicling the history of botanical exploration of the area. Based on the experience of the project, recomendations are made about procedures of inventory of plant species in tropical forest.

Key words: Central Amazon, tropical rain forest, floristics.

\section{RESUMO}

(Flora da Reserva Ducke, Amazônia Central, Brasil) A Reserva Ducke na Amazônia central foi o alvo de um estudo florístico intensivo entre 1992 e 1999. O projeto mostrou que a biodiversidade na Reserva era bem maior do que havia sido estimada anteriormente. São apresentados dados ilustrando a história de exploração botânica na área. Baseando-se na experiência do projeto, são feitas recomendações sobre procedimentos para realização inventário de espécies vegetais em floresta tropical.

Palavras chave: Amazônia Central, floresta de terra-firme, florística.

\section{INTRODUÇÃO}

A Reserva Florestal Adolfo Ducke é uma área de floresta amazônica primária de 100 $\mathrm{km}^{2}$, localizada próxima à cidade de Manaus e pertence ao Instituto Nacional de Pesquisas da Amazônia (INPA) (fig. 1). Foi declarada como Reserva Biológica em 1963, nesta época a cidade de Manaus possuía uma população de aproximadamente 40.000 habitantes. Nas últimas quatro décadas (até 2005) a população saltou para cerca de 2.000.000 de habitantes e a cidade expandiu sua área urbanizada, chegando aos limites da Reserva em duas de suas laterais. Aliado a isso, a devastação da floresta nas áreas próximas às outras duas extremidades, vem transformando a Reserva em um fragmento florestal isolado. Apesar dessas interferências nos seus arredores, os recursos naturais da Reserva Ducke continuam bem preservados, muito embora a área seja periodicamente invadida por caçadores de animais silvestres e outras pessoas interessadas na extração de produtos florestais.
O nome da Reserva é uma homenagem ao botânico Adolfo Ducke (1875-1969), que foi um dos pesquisadores mais ativos no estudo da flora amazônica durante a maior parte do século XX. Este ilustre botânico nasceu em Trieste, então parte do Império AustroHúngaro, e migrou para Belém em (1899) a convite de Emílio Goeldi, que era o Diretor do então Museu Paraense. Em 1918 ele foi contratado pelo Jardim Botânico do Rio de Janeiro. Ducke realizou um intenso e amplo trabalho de campo na região amazônica em expedições realizadas pelo Museu Paraense, Instituto Agronômico do Norte (atualmente Embrapa Amazônia Oriental) e pelo Jardim Botânico do Rio de Janeiro. Visitou Manaus inúmeras vezes entre 1910-1953 e a maioria de suas coleções desta região foi feita entre 1941-1946, incluindo coletas em locais hoje totalmente desmatados e urbanizados. É difícil confirmar se ele, de fato, coletou na área da Reserva que hoje tem seu nome, provavelmente não, uma vez que não consta nas suas etiquetas os nomes dos igarapés da Reserva. 


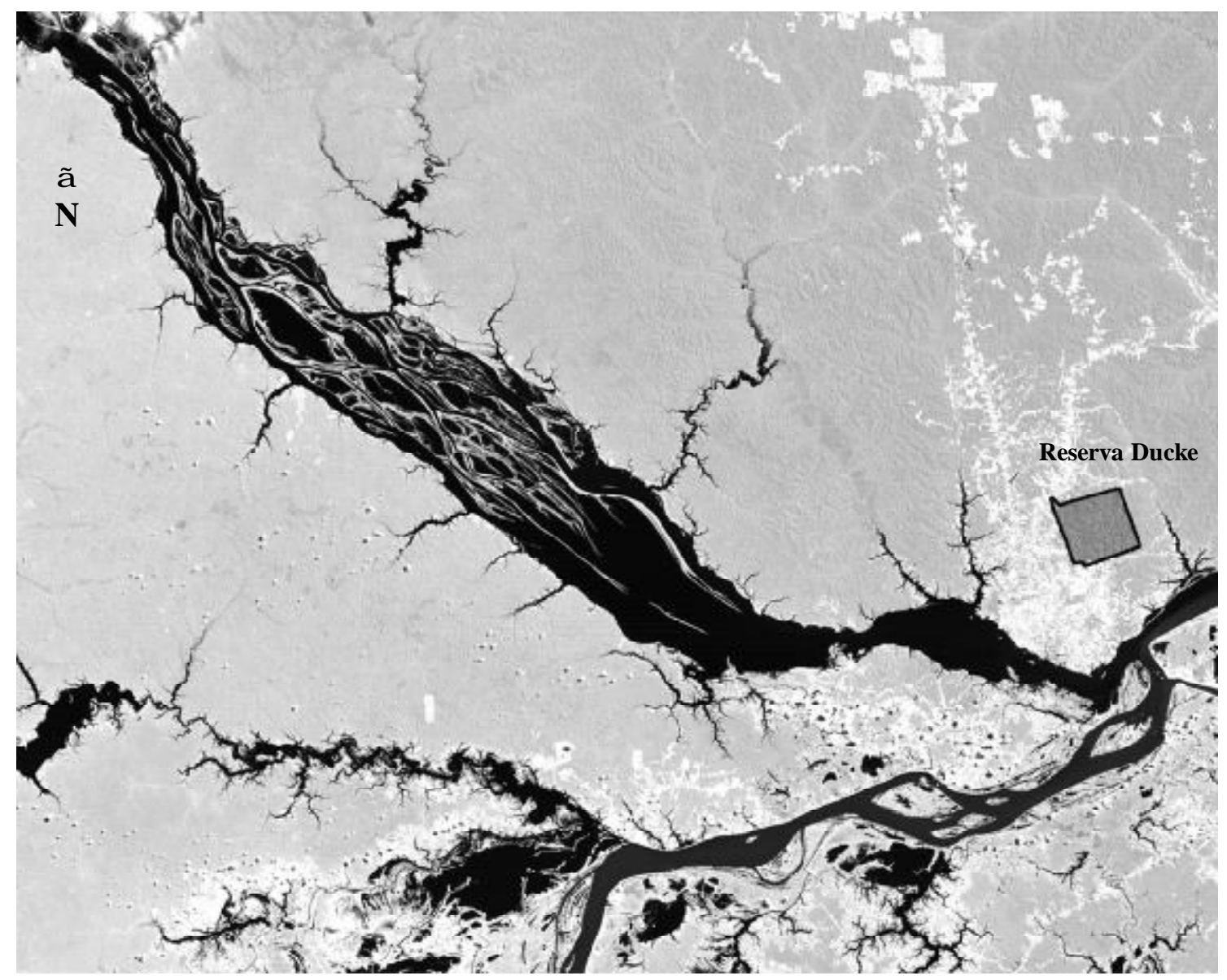

Figura 1 - Localização da Reserva Ducke. A cidade de Manaus é indicada pela área clara ao sul e leste. As duas estradas principais saindo da cidade (BR174, Manaus - Caracaraí, ao norte, e o AM010, Manaus - Itacoatiará a oeste) são visíveis. A Reserva fica perto dos dois grandes rios, o Negro, acima e o Solimões de baixo. (Imagen Landsat de 1995, fonte INPE).

\section{O histórico do Projeto Flora da Reserva Ducke}

A idéia de se publicar a flora da Reserva Ducke foi concebida na década de 1980 por Ghillean T. Prance, na época Diretor do New York Botanical Garden, Marlene Freitas da Silva e William A. Rodrigues, então pesquisadores do INPA. Em 1990, Prance se mudou para a Inglaterra e assumiu a Diretoria do Royal Botanic Gardens, Kew e obteve o financiamento para o desenvolvimento do projeto. O auxílio financeiro foi concedido pelo governo britânico, através da Overseas Development Agency (ODA), atualmente conhecida como Department for International Development (DFID). O projeto foi chamado "Projeto Flora e Vegetação da Amazônia Central", mas logo ficou conhecido como "Projeto Flora da Reserva Ducke (PFRD)".

As atividades do projeto foram iniciadas com a contratação do botânico José Eduardo L.S. Ribeiro, sob administração de Bruce W. Nelson (pesquisador do INPA) e coordenação de Maria Lúcia Absy, Diretora da Coordenação de Pesquisas em Botânica do INPA naquela época. Em 1993, fui contratado para coordenar os assuntos internacionais do projeto e seu início se concretizou. O financiamento obtido inicialmente cobriria a execução do projeto por três anos e depois foi extendido por mais dois. Entretanto, as atividades do projeto estenderamse até 1999. No planejamento, foram previstos quatro produtos principais: um check-list da Flora da Reserva, o tratamento taxonômico para todas as famílias, um guia prático ao nível 
de família, além de mapas e publicações sobre a flora da Amazônia Central.

O check-list está disponível no site http:/ /www.inpa.gov.br/projetos/ducke/. A parte de vegetação ficou na coordenação de Bruce Nelson, e foi completada em 1996. Os tratamentos taxonômicos para cada família estão sendo publicados na Rodriguésia, sendo este o primeiro fascículo. O guia foi publicado em 1999 (Ribeiro et al. 1999) e seu conteúdo vai até o nível de espécie.

Em review, o guia recebeu avaliações boas incluindo "... the authors have started something that should quickly accelerate knowledge of the plant resources of the Amazon region ...” (Foster 2000), “... this book excels in a number of important criteria, such as high information content, richness of graphics, full color pages, high degree of innovation, reasonable price ...” (Berry 2000) “... sets a completely new standard for plant identification manuals in the tropics ..." (Rejmánek \& Brewer 2001).

\section{Amazônia central e SeU CONHECIMENTO BOTÂNICO}

A planície do rio Amazonas inclui uma vasta área de aproximadamente $5.000 .000 \mathrm{de}$ $\mathrm{km}^{2}$, abrangendo o território de seis países sulamericanos. A formação vegetal dominante é mata de terra firme, termo aplicado na Amazônia para designar a floresta não alagada periodicamente. Encontram-se também, dentro dos limites da planície do rio Amazonas, as matas de várzea, que são áreas da floresta que são periodicamente inundadas e, ainda formações mais abertas, distribuídas de forma esparsa, sobre solo arenoso, que são as campinas e campinaranas. A cidade de Manaus localiza-se na confluência dos dois maiores rios da bacia amazônica, o rio Solimões, rico em sedimentos erodidos das montanhas andinas mais jovens, e o rio Negro, que drena a planície mais antiga ao norte e quase não possui sedimentos. A diferença nas águas destes dois rios determina o estabelecimento de diferentes floras: tipo igapó, inundada por água "preta" com menor quantidade de sedimentos, e várzea, inundada por águas "claras" e ricas em sedimentos. Da mesma forma, o tipo de solo influencia fortemente a composição florística local.

Os primeiros coletores botânicos só visitaram a Amazônia Central no século XVIII. Antes de 1860 somente seis coletores botânicos haviam visitado a área (Urban 1906): Alexandre Rodrigues Ferreira (1784-88), Karl Friedrich Phillip von Martius (1819-20), Ludwig Riedel (1828), Eduard Friedrich Poeppig (183132), Alfred Russel Wallace (1849-52) e Richard Spruce (1850-55). Grande parte destas coleções históricas estão depositadas em herbários estrangeiros. A Flora brasiliensis, (publicada em 40 volumes entre 1840 e 1906) contou com o pouco material coletado nestas expedições. Diante disto, muitas espécies da Amazônia Central com distribuições mais amplas foram descritas a partir de coleções feitas em outros países, notadamente através das viagens de botânicos como Moritz Richard e Robert Herman Schomburgk, Jean Baptiste Christophe Fuseé Aublet, Hipolito Ruíz e José Pavon e Baron Alexander von Humboldt e Aime Bonpland.

No século XX foram criados os primeiros herbários na Amazônia, incluindo o do Museu Paraense Emílio Goeldi em Belém (MG), e do Jardim Botânico de Manaus (coleção perdida). Também foram realizadas expedições do Jardim Botânico do Rio de Janeiro, resultando em importantes coleções de Jacques Huber, João Geraldo Kuhlmann, Dimitri Sucre, e especialmente Adolfo Ducke. Ducke coletou amplamente na Amazônia, freqüentemente revisitando árvores previamente coletadas com flores para obter frutos da mesma fonte, uma prática que foi repetida durante o PFRD.

Em meados do século XX foram fundados os herbários do IAN (Instituto Agronômico do Norte) em 1940 e INPA em 1954. Os principais coletores destes herbários, Ducke, João Murça Pires, Ricardo Lemos Fróes, Emanuel de Oliveira e George Alexander Black do IAN, e William Antônio Rodrigues do INPA coletaram na Amazônia Central, e Rodrigues concentrou suas coletas na região da Reserva Ducke e na estrada Manaus-Itacoatiara (AM-010). 


\section{Coletas botânicas na Reserva Ducke}

Antes do início do Projeto Flora da Reserva Ducke (PFRD), foi feito um levantamento do material botânico proveniente da Reserva e que se encontram depositado no Herbário do INPA. Este inventário indicou que pouco mais de 7.000 amostras haviam sido coletadas dentro dos limites da Reserva. Grande parte deste material eram coletas provenientes dos estudos realizados por William Rodrigues (hoje pesquisador aposentado do INPA) e seu auxiliar de campo Osmarino P. Monteiro. Essas amostras foram coletadas entre os anos de 1957-1966, a partir de árvores marcadas em uma área de cerca de 16 ha. Algumas áreas desses parcelas foram destruídas quando da alteração no percurso da estrada de acesso à Reserva e, conseqüentemente, o projeto foi abandonado. Rodrigues e Monteiro foram os responsáveis por cerca de $50 \%$ das coletas feitas na Reserva Ducke antes de 1993 (tabela 1). A maioria dessas coleções é de material fértil.

Também existem no herbário do INPA várias amostras estéreis oriundas da área da Reserva, que são o resultado dos estudos fitossociológicos lá realizados. Este material é, em grande parte, inadequado para os estudos taxonômicos e até $2002,10 \%$ estava identificados somente até o nível de família e $30 \%$ até gênero.

Prance (1990) registrou para a Reserva um total de 825 espécies de plantas vasculares. Seu levantamento foi baseado nos dados do Programa Flora. Também estimou que com identificações mais apuradas este número poderia ser elevado para aproximadamente 1.030 espécies. Após a informatização da coleção da Reserva Ducke depositada no herbário do INPA, Ribeiro et al. (1994) estimaram a ocorrência em 1199 espécies.

\section{Caracterização dos ambientes encontrados na Reserva Ducke}

A vegetação principal na Reserva é mata de terra firme. As matas de várzeas e de igapós não estão presentes. Estes tipos de vegetação não aparecem porque os rios dentro da Reserva não transbordam regularmente como ocorre nos principais rios da Amazônia. Também não são encontradas na Ducke as formações de Campinas, que são áreas com árvores baixas e esparsas sobre solo arenoso.

São encontrados quatro tipos de ambientes na Reserva, de acordo com a classificação geral de mata de terra firme e estes são definidos pelo tipo de relevo e composição do solo. Em geral, os solos nas partes mais altas são latossolos amarelo-álicos, argilosos e, nas partes mais baixas são podsólicos arenosos.

As florestas de platô, como o próprio nome sugere, estão situadas nas áreas mais altas e planas da Reserva. O solo nessas áreas é argiloso, bem drenado e pobre em nutrientes. Neste tipo de floresta são encontradas as maiores árvores e, provavelmente, as mais antigas. $\mathrm{O}$ dossel atinge entre 30-40 metros de altura, com algumas árvores emergentes chegando a 5060 metros de altura. Dentre as emergentes destacam-se as Leguminosae, como Dinizia excelsa Ducke e Pseudopiptadenia

Tabela 1 - Principais coletores de material fértil na Reserva Ducke antes de 1993.

\begin{tabular}{lc}
\hline Coletor & No $^{\text {coletas }}$ \\
\hline Rodrigues, W.A. & 1577 \\
Souza, J.A. ${ }^{1}$ & 308 \\
Prance, G.T. & 216 \\
Ferreira, E. & 118 \\
Loureiro, A. & 115 \\
Monteiro, O.P. & 73 \\
Coêlho, D.F. & 73 \\
Silva, M.F. da & 60 \\
Albuquerque, B.W.P. & 59 \\
Coêlho, L.F. & 67 \\
Mello, F. & 55 \\
Nascimento, J.R. & 46 \\
Gentry, A.H. & 42 \\
Küchmeister, H.E.C. & 40 \\
Outros & 539 \\
Total & $\mathbf{3 3 4 8}$
\end{tabular}

${ }^{1}$ Geralmente "Aluísio" nas etiquetas; ${ }^{2}$ geralmente "Osmarino" nas etiquetas.

Rodriguésia 56 (86): 9-25. 2005 
psilostachya (DC.) G.P. Lewis \& M.P. Lima, e Lecythidaceae, como as espécies de Lecythis e Cariniana micrantha Ducke. O sub-bosque é dominado por palmeiras acaules, especialmente Attalea atteleiodes (Barb. Rodr.) Wess. e Astrocaryum sciophilum (Miq.) Pulle.

As florestas de baixio ocorrem ao longo dos igarapés, nas áreas mais baixas. $\mathrm{O}$ solo é arenoso, muito úmido e encharcado nas épocas de maior pluviosidade. Muitas árvores possuem raízes superficiais ou escoras e, algumas, com pneumatóforos. O dossel é mais baixo do que nas regiões de platôs, com 25-30 metros de altura, e com muitas palmeiras, como Oenocarpus bataua Mart. e Mauritia flexuosa L.f. No sub-bosque podem ser encontradas ervas de porte relativamente grande das famílias Marantaceae, Rapateaceae, Cyclanthaceae e a palmeira acaule Attalea microcarpa Mart.

As florestas de vertente ocorrem nas inclinações dos platôs. Os solos dessas florestas são mais arenosos nas porções mais baixas. A comunidade vegetal e a altura do dossel são similares aos das florestas de platô. Entretanto, a quantidade de árvores emergentes é bem menor. A floresta de vertente pode ser considerada um tipo de transição entre a de baixio e a de platô. Cabe ressaltar a transição entre vertente e baixio é muito mais abrupta que entre vertente e platô.

As florestas de campinarana são encontradas nas planícies próximas dos igarapés e nessas áreas o solo é arenoso e com grande quantidade de serrapilheira. As árvores que ocorrem nessas florestas possuem menor DAP que aquelas dos platôs. O dossel também é mais baixo, geralmente entre 15-25 metros de altura. As espécies que crescem nessas formações são típicas e entre elas está Aldina heterophylla Spruce ex Benth., uma árvore freqüente do dossel que possui seus ramos cobertos com epífitas. As epífitas são especialmente abundantes nas campinaranas e são raras nos outros ambientes. O sub-bosque tem relativamente poucas palmeiras e é dominado pelas Marantaceae.
As áreas alteradas por ação antrópica são poucas na Reserva e estão localizadas principalmente nos arredores da sede e na estrada de acesso.

\section{Metodologia de coleta do PFRD}

Foi constatado no final de 1993 que, provavelmente, ocorreriam muito mais espécies na área da Reserva do que as previsões iniciais indicavam e do que já havia sido coletadas efetivamente. Com o intuito de sanar este problema, optou-se por ampliar o escopo do guia ilustrado e este passou a ser no nível de espécie e deveria abranger todas as plantas vasculares. Foi dado enfoque maior para as características vegetativas das espécies. Inicialmente o guia previa apoiar identificação até família. O resultado desta mudança na concepção do projeto incentivou os coletores a buscarem exemplares vivos de todas as espécies para que pudessem ser fotografadas para o guia. A busca inicial dessas espécies no campo foi feita a partir das informações contidas nas etiquetas dos materiais de herbário.

Uma equipe formada de três auxiliares de campo (Paulo A.C.L. Assunção, Everaldo C. Pereira e Cosmo F. da Silva), com dedicação integral ao projeto, às vezes acrescida de mais três ajudantes, foi contratada para trabalhar permanentemente na Reserva. Vários especialistas botânicos foram convidados a integrar a equipe do projeto. Alguns deles participaram dos trabalhos de campo na Reserva (tabela 2) e outros não. Porém todos receberam o material coletado para identificação e elaboração das monografias. Seis jovens botânicos brasileiros (José Eduardo L. da Silva Ribeiro, Alberto Vicentini, Cynthia A. Sothers, Maria A. da Silva Costa, Maria A. D. de Souza e Joneide M. de Brito) foram contratados para ajudar nas atividades do projeto, principalmente aquelas envolvendo o trabalho de campo (coleta de material) junto com os mateiros e especialistas, identificação das espécies e ajuda na elaboração do guia. Quatro alunas também participaram por longo tempo nas atividades do projeto: 
Lúcia H.P. Martins, Lúcia G. Lohmann, Mariana R. Mesquita e Lílian C. Procópio. Inicialmente, cada botânico integrante do projeto ficou responsável por um grupo de famílias. Estes botânicos analisaram as coletas previamente depositadas no herbário do INPA, prepararam uma pasta com fotocópias das amostras e informações da literatura. Após esta etapa, cada botânico, sempre acompanhado por um dos mateiros, seguiu para o campo em busca das espécies na natureza. As amostras foram coletadas, descritas resumidamente e fotografadas para elaboração do guia. No caso

Tabela 2 - Relação de especialistas que visitaram a Reserva Ducke (1993 - 1999) - e seus respectivos herbários na época.

\begin{tabular}{|c|c|}
\hline Visitante & Instituição \\
\hline Acevedo, Pedro & US \\
\hline Amaral, Maria do C. & UEC \\
\hline Berg, Cornelius C. & $\mathrm{BG}$ \\
\hline Bittrich, Volker & UEC \\
\hline Campos, Marina T.V. do A. & $\mathrm{SPF}$ \\
\hline Cordeiro, Inês & $\mathrm{SP}$ \\
\hline Daly, Douglas C. & NY \\
\hline Esteves, Gerlene & SP \\
\hline Forzza, Rafaela & SPF \\
\hline Gomes, Fabiana P. & SPF \\
\hline Harley, Ray & $\mathrm{K}$ \\
\hline Henderson, Andrew & NY \\
\hline Kawasaki, Maria Lúcia & SP \\
\hline Lima, Haroldo C. de & $\mathrm{RB}$ \\
\hline Lima, Rita B. & SPF \\
\hline Malta, Lilian & SPF \\
\hline Maas, Hiltje & $\mathrm{U}$ \\
\hline Mass, Paul J.M. & $\mathrm{U}$ \\
\hline Mayo, Simon & $\mathrm{K}$ \\
\hline Mori, S.M. & NY \\
\hline Nee, Michael H. & NY \\
\hline Nicolau, Sueli & $\mathrm{SP}$ \\
\hline Oliveira, Alexadre A. & SPF \\
\hline Pennington, T.D. & $\mathrm{K}$ \\
\hline Prado, Jefferson & $\mathrm{SP}$ \\
\hline Pirani, José Rubens & SPF \\
\hline Prance, Ghillian T. & $\mathrm{K}$ \\
\hline Renner, Suzanne & MO \\
\hline Rodrigues, William A. & UPCB \\
\hline Rossi, Lúcia & SP \\
\hline Secco, Ricardo & $\mathrm{MG}$ \\
\hline Werff, Henk van der & MO \\
\hline
\end{tabular}

de árvores, alguns indivíduos de cada espécie foram plaqueados e mapeados para facilitar a sua localização posterior, visando à realização de futuras coletas de flores e frutos. Em alguns casos, vários dos estudantes ligados com o projeto foram incentivados pesquisar um grupo em particular. Vários pesquisadores do INPA também participaram do projeto: Aldaléa $\mathrm{S}$. Tavares, José M.S. Miralha, Maria das Graças G. Vieira, Carlos A.A. de Freitas, Maria de Lourdes Soares e José Augusto da Silva.

Aproximadamente 5.000 plantas lenhosas (árvores, arvoretas e lianas) foram plaqueadas ao longo das trilhas na Reserva. Essas plantas foram mapeadas e suas localizações podem ser facilmente encontradas nos mapas das trilhas que foram confeccionados. Esses mapas estão disponíveis para consulta no site do PFRD. Também há um banco de dados dessas plantas no Laboratório de Taxonomia Botânica (CPBO) do INPA. As plantas marcadas encontram-se principalmente na porção noroeste da Reserva, que fica entre a entrada e o igarapé Acará. Além desta área, outras, como as regiões dos igarapés Tingá, no sudoeste, Água Branca, no nordeste, e Ipiranga, no sudoeste, foram as mais visitadas para coleta de material.

Com um planejamento arrojado de coleta, foi possível dobrar o número de coleções (tabela 3) e triplicar o número de amostras férteis para a área da Ducke.

Quando possível, uma duplicata de cada espécie foi doada para o herbário da instituição do especialista; as unicatas foram emprestadas. Após o término da elaboração da monografia da família as unicatas foram devolvidas para o herbário do INPA. Assim que as identificações chegavam eram repassadas para as duplicatas ainda existentes e que posteriormente foram distribuídas para outros herbários no Brasil e no exterior. O herbário do Royal Botanic Gardens, Kew (K) e o Museu Goeldi (MG) também receberam coleções completas, exceto no caso das unicatas. O controle da distribuição das coleções para os herbários foi registrado no banco de dados do projeto e este foi utilizado 
para gerar as listas de exsicatas citadas nos tratamentos taxonômicos. Os principais herbários que receberam duplicatas em ordem de prioridade (além do INPA, K e MG) foram:

RB - Jardim Botânico de Rio de Janeiro NY - Jardim Botânico de Nova Iorque SP - Instituto Botânico, São Paulo MO - Jardim Botânico de St. Louis, Missouri IAN - Embrapa Amazônia Oriental, Belém U - Universidade de Utrecht, Holanda.

No final do projeto a coleção de referência foi doada para herbários amazônicas. Em ordem de prioridade foram:

SAPECA - Sociedade de Pesquisas e Conservação da Amazônia

HUAM - Universidade Federal de Amazonas

PRANCE - Universidade Luterana do Brasil, Manaus

UFAC - Universidade Federal de Acre

HAMAB - Instituto Estadual de Pesquisas do Amapá

UFMT - Universidade Federal de Mato Grosso

MIRR - Universidade Federal de Roraima.

\section{Plano geral dos}

\section{TRATAMENTOS TAXONÔMICOS}

Conforme referido anteriormente, na concepção original do projeto foi prevista a publicação dos tratamentos taxonômicos de cada família para a Reserva. A lista dos especialistas envolvidos e suas respectivas famílias de especialidade estão apresentados na tabela 4. Algumas famílias vultosas (p. ex., Leguminosae e Euphorbiaceae) foram monografadas por diferentes autores e serão apresentadas em partes separadas. Os tratamentos serão publicados em volumes da Revista Rodriguésia de acordo com a ordem de entrega dos manuscritos ao corpo editorial da revista. Este procedimento foi adotado para que os autores que já entregaram os trabalhos não fiquem prejudicados. Desta forma, não seguiremos ordem alfabética ou sistema de classificação.
Tabela 3 - Principais coletores de material fértil na Reserva Ducke (1992 - 1999).

\begin{tabular}{lc}
\hline Coletor & No $^{\circ}$ coletas \\
\hline Ribeiro, J.E.L.S. & 1167 \\
Sothers, C.A. & 969 \\
Vicentini, A. & 880 \\
Assunção, P.A.C.L. & 855 \\
Costa, M.A.S. da & 762 \\
Souza, M.A.D. de & 578 \\
Nascimento, J.R. & 287 \\
Hopkins, M.J.G. & 245 \\
Soares, M.L. & 180 \\
Campos, M.T.V. do A. & 147 \\
Prado, J. & 142 \\
Santos, J.L. & 132 \\
Leme, C.D. & 117 \\
Martins, L.H.P. & 93 \\
Pruski, J.F. & 84 \\
Ramos, J.F. & 76 \\
Coêlho, D.F. & 73 \\
Brito, J.M. de & 61 \\
Matteo, B.C. & 58 \\
Lohmann, L.G. & 57 \\
Miralha, J.M.S. & 47 \\
Gomes, F.P. & 37 \\
Forzza, R.C. & 36 \\
Cordeiro, I. & 32 \\
Mesquita, M.R. & 30 \\
Nee, M. & 27 \\
Procópio, L.C. & 26 \\
Outros & 150 \\
Total & $\mathbf{7 3 4 8}$ \\
\hline
\end{tabular}

Um índice de famílias será apresentado em cada novo volume que for publicado, para facilitar a localização das famílias nos volumes.

Adotaram-se as famílias segundo Cronquist (1981), exceto Leguminosae (Fabaceae s.l.) que é tratado como três subfamílias (Mimosoideae, Caesalpinioideae, e Papilionoideae). Foi adotado um formato geral para os tratamentos, porém sem um estilo padronizado rigoroso. Os autores tiveram uma certa flexibilidade sobre a inclusão de outras espécies regionais, nível de detalhamento das descrições e na elaboração de chaves. Cada 
tratamento inclui: bibliografia relevante para a família; descrição da família; chave para identificação dos gêneros (em alguns tratamentos as chaves conduzem à identificação dos gêneros e espécies simultaneamente); descrições breves dos gêneros; chaves para identificação das espécies. Para cada espécie são apresentadas as autorias, obra princeps, principais sinônimos, nome popular local (caso haja) e descrições. Apresentam-se também comentários sobre distribuição geográfica, hábitats de ocorrência e fenologia. A citação do material examinado inclui: coletor e número, data de coleta e estado fenológico e herbários onde está depositado o material. O local específico de coleta é citado somente para coleções provenientes de locais fora da Reserva.

Por limitações orçamentárias não foi possível confecçionar muitas pranchas originais. Em muitos tratamentos os autores utilizaram ilustrações já publicadas em outras obras ou providenciou as pranchas com financiamento próprio.

A publicação dos tratamentos tem sido muito demorada. A causa disto consiste na falta de um meio de publicação e de financiamento. Uma vez que não houve a publicação durante o projeto, o financiamento previsto para isso foi retirado. Nos anos seguintes vários outros meios de publicação foram buscados, mas sem resultar em um financiamento adequado até a iniciativa do Corpo Editorial da Rodriguésia. Com a publicação destes primeiros tratamentos e de outros já submetidos aos próximos fascículos da Rodriguésia, espero que os tratamentos que não nos foram entregues sejam logo submetidos.

\section{DIVERSIDADE DE ESPÉCIES na Reserva Ducke}

A diversidade registrada para a Reserva Ducke foi de 2.079 espécies. A maioria das espécies pertence ao grupo das arbóreas, com $54 \%$, seguida pelas lianas (14\%), ervas (10\%), epífitas (8\%), arbustos (7\%), hemiepífitas (4\%), palmeiras $(2 \%)$, saprófitas $(1 \%)$ e parasitas $(1 \%)$. Dentre as famílias com representantes predominantemente arbóreos destacam-se: Leguminosae, Lauraceae, Sapotaceae, Chrysobalanaceae, Moraceae, Burseraceae, Lecythidaceae, Apocynaceae e Myristicaceae. Myrtaceae e Annonaceae, são as mais diversas no subdossel (tabela 5).

No sub-bosque, Rubiaceae, Piperaceae e Melastomataceae dominam entre as plantas lenhosas. Dentre as ervas as mais diversas são espécies de pteridófitas e Marantaceae. Poaceae e Cyperaceae têm relativamente poucas espécies, e ocorrem principalmente em áreas alteradas.

Dentre as lianas, a maior diversidade é encontrada em Bignoniaceae. Há ainda um grande número de famílias que apresentam um menor número de espécies lianas incluindo Leguminosae (especialmente Machaerium e Bauhinia), Apocynaceae, Convolvulaceae, Cucurbitaceae, Dilleniaceae, Hippocrateaceae, Malpighiaceae, Menispermaceae, Passifloraceae, Polygalaceae e Sapindaceae. Dentre as epífitas, Orchidaceae é a maior família com cerca de 85 espécies epífitas, seguida por Bromeliaceae (ca. 10 spp.) e Araceae (ca. 8 spp.). Dentre as pteridófitas, cerca de 45 espécies são epífitas. Vale ressaltar, que as campinaranas são extremamente ricas em epífitas em comparação com os outros ambientes encontrados na Reserva. 
Tabela 4 - As famílias tratadas na Flora da Reserva Ducke (tratamentos publicados neste volume ou já submetidos para publicação estão em negrito).

\begin{tabular}{|c|c|c|c|c|}
\hline $\begin{array}{l}\text { GRUPO } \\
\text { Família } \\
\end{array}$ & Especialistas $^{1}$ & Gêneros & Táxons $^{2}$ & Novas $^{3}$ \\
\hline \multicolumn{5}{|l|}{ PTERIDÓFITAS } \\
\hline Aspleniaceae & J. Prado & 1 & 4 & \\
\hline Blechnaceae & J. Prado & 1 & 1 & \\
\hline Cyatheaceae & J. Prado \& C.A.A. Freitas & 1 & 3 & \\
\hline Davalliaceae & J. Prado & 2 & 5 & \\
\hline Dennstaedtiaceae & J. Prado & 3 & 6 & \\
\hline Dryopteridaceae & C.A.A. Freitas \& J. Prado & 2 & 3 & \\
\hline Gleicheniaceae & J. Prado & 2 & 2 & \\
\hline Grammatidaceae & J. Prado & 2 & 3 & \\
\hline Hymenophyllaceae & P. Windisch & 2 & 11 & \\
\hline Lomariopsidaceae & J. Prado & 2 & 9 & \\
\hline Lycopodiaceae & C.A.A. Freitas \& P. Windisch & 1 & 1 & \\
\hline Marattiaceae & J. Prado & 1 & 3 & \\
\hline Metaxyaceae & M.A.S. da Costa \& J. Prado & 1 & 1 & \\
\hline Ophioglossiaceae & M.A.S. da Costa \& J. Prado & 1 & 1 & \\
\hline Polypodiaceae & J. Prado & 6 & 9 & \\
\hline Pteridaceae & J. Prado & 3 & 6 & \\
\hline Schizaeaceae & J. Prado & 3 & 5 & \\
\hline Selaginellaceae & J. Prado \& C.A.A. Freitas & 1 & 6 & \\
\hline Tectariaceae & J. Prado & 1 & 1 & \\
\hline Thelypteridaceae & J. Prado & 1 & 1 & \\
\hline \multirow[t]{2}{*}{ Vittariaceae } & J. Prado \& P. Labiak & 3 & 6 & \\
\hline & Total Pteridófitas & 40 & 87 & $\mathbf{0}$ \\
\hline \multicolumn{5}{|l|}{ GIMNOSPERMAS } \\
\hline Zamiaceae & A. Vicentini & 1 & 1 & \\
\hline \multirow[t]{2}{*}{ Gnetaceae } & A. Vicentini & 1 & 4 & \\
\hline & Total Gimnospermas & 2 & 5 & $\mathbf{0}$ \\
\hline \multicolumn{5}{|l|}{ DICOTILEDÔNEAS } \\
\hline Acanthaceae & C. Kameyama & 5 & 7 & \\
\hline Amaranthaceae & L.G. Lohmann \& M.A.S. da Costa & 2 & 2 & \\
\hline Anacardiaceae & J. Mitchell & 5 & 8 & \\
\hline Anisophyllaceae & G.T. Prance & 1 & 1 & \\
\hline \multirow[t]{2}{*}{ Annonaceae } & P.J.M. Maas, H. Maas & 16 & 59 & 4 \\
\hline & \& J.M.S. Miralha & & & \\
\hline Apiaceae & J.E.L.S. Ribeiro & 1 & 1 & \\
\hline Apocynaceae & A.A. Oliveira & 15 & 41 & \\
\hline Aquifoliaceae & A. Vicentini & 1 & 1 & \\
\hline Araliaceae & D. Frodin \& L. Malta & 2 & 3 & 1 \\
\hline Aristolochiaceae & J.E.L.S. Ribeiro & 1 & 3 & \\
\hline Asclepiadaceae & A.M. Farinaccio & 2 & 4 & \\
\hline Asteraceae & J.E.L.S. Ribeiro & 12 & 14 & \\
\hline Bignoniaceae & L.G. Lohmann & 22 & 54 & \\
\hline Bombacaceae & G.L. Esteves & 8 & 13 & \\
\hline Boraginaceae & G.T. Prance & 1 & 11 & \\
\hline
\end{tabular}

${ }^{\overline{1}}$ Principais pessoas responsáveis pelas identificações. No caso de famílias ainda não submetidas, o autor do tratamento poderá vir a ser diferente. ${ }^{2}$ Espécies, variedades ou subespécies. ${ }^{3}$ Número de novos táxons confirmados. ${ }^{4}$ Tratamento da família submetido ou publicado parcialmente. 


\begin{tabular}{|c|c|c|c|c|}
\hline Família & Especialistas $^{\top}$ & Gêneros & Táxons $^{2}$ & Novas \\
\hline Burseraceae & D.C. Daly & 5 & 42 & 2 \\
\hline Cactaceae & J.E.L.S. Ribeiro & 1 & 1 & \\
\hline Capparaceae & M.A.S. da Costa \& L.G. Lohmann & 2 & 2 & \\
\hline Caryocaraceae & G.T. Prance \& M.F. da Silva & 1 & 4 & \\
\hline Caryophyllaceae & L.G. Lohmann \& M.A.S. da Costa & 1 & 1 & \\
\hline Cecropiaceae & C.C. Berg \& J.E.L.S. Ribeiro & 3 & 24 & \\
\hline Celastraceae & J.M. de Brito & 2 & 3 & \\
\hline Chrysobalanaceae & G.T. Prance & 5 & 53 & 2 \\
\hline Clusiaceae & V.Bittrich & 14 & 49 & \\
\hline Combretaceae & N.M.F. da Silva \& M. da C. Valente & 2 & 7 & \\
\hline Dilleniaceae & C.A. Sothers & 4 & 14 & \\
\hline Duckeodendraceae & M. Nee & 1 & 1 & \\
\hline Ebenaceae & C.A. Sothers & 1 & 7 & 1 \\
\hline Elaeocarpaceae & A. Vicentini & 1 & 17 & \\
\hline Eremelopidaceae & B. Stannard & 1 & 1 & \\
\hline Ericaceae & J.E.L.S. Ribeiro & 1 & 1 & \\
\hline Erythroxylaceae & G.T. Prance & 1 & 3 & \\
\hline Euphorbiaceae $^{4}$ & R. de S. Secco & 27 & 50 & \\
\hline Flacourtiaceae & S. Smartzy & 4 & 17 & \\
\hline Gentianaceae & H. Maas \& P.J.M. Maas & 4 & 7 & \\
\hline Gesneriaceae & A. Chautems & 5 & 8 & \\
\hline Hernandiaceae & C.A. Sothers & 1 & 1 & \\
\hline Hippocrateaceae & J.E.L.S. Ribeiro & 7 & 14 & \\
\hline Hugoniaceae & R. de S. Secco & 2 & 2 & \\
\hline Humiriaceae & M. Wenzel \& G.T. Prance & 5 & 10 & \\
\hline Icacinaceae & J.M. de Brito & 6 & 8 & \\
\hline Lacistemataceae & M. Nee & 1 & 3 & \\
\hline Lamiaceae & R.M. Harley & 2 & 3 & \\
\hline Lauraceae & H. van der Werff \& A. Vicentini & 13 & 100 & 10 \\
\hline Lecythidaceae & S.M. Mori & 7 & 38 & 3 \\
\hline \multicolumn{5}{|l|}{ Leguminosae: } \\
\hline Caesalpinioideae & A.S. Tavares e M.F. da Silva & 17 & 54 & 1 \\
\hline Mimosoideae $^{4}$ & $\begin{array}{l}\text { M.G.G. Vieira, T.D. Pennington } \\
\text { \& L.C. Procópio }\end{array}$ & 16 & 68 & 1 \\
\hline Papilionoideae ${ }^{4}$ & H.C. da Lima \& M.R. Mesquita & 22 & 66 & \\
\hline Lentibulariaceae & M.A.S. da Costa & 1 & 1 & \\
\hline Loganiaceae & D. Zappi & 4 & 10 & \\
\hline Loranthaceae & B. Stannard & 5 & 9 & \\
\hline Lythraceae & T. Cavalcante & 1 & 2 & \\
\hline Malpighiaceae & W.R. Anderson & 10 & 24 & \\
\hline Malvaceae & G.L. Esteves & 2 & 2 & \\
\hline Marcgraviaceae & J.E.L.S. Ribeiro & 4 & 5 & \\
\hline Melastomataceae & S.S. Renner & 13 & 58 & \\
\hline Meliaceae & T.D. Pennington & 3 & 23 & \\
\hline Memecylaceae & S.S. Renner & 1 & 12 & \\
\hline Menispermaceae & R. Ortiz-Gentry \& C. Ott & 8 & 15 & \\
\hline Monimiaceae & S.S. Renner & 1 & 1 & \\
\hline Moraceae & C.C. Berg \& J.E.L.S. Ribeiro & 11 & 47 & 1 \\
\hline Myristicaceae & W.A. Rodrigues & 4 & 25 & \\
\hline Myrsinaceae & J.J. Pipoly & 3 & 10 & \\
\hline Myrtaceae & M.L. Kawasaki \& B.K. Holst & 7 & 65 & 1 \\
\hline
\end{tabular}




\begin{tabular}{|c|c|c|c|c|}
\hline Família & Especialistas $^{1}$ & Gêneros & Táxons $^{2}$ & Novas \\
\hline Nyctaginaceae & J.E.L.S. Ribeiro & 2 & 7 & \\
\hline Ochnaceae & M. de C. Amaral & 3 & 9 & \\
\hline Olacaceae & L. Rossi & 7 & 11 & \\
\hline Onagraceae & $\begin{array}{l}\text { L.G. Lohmann, M.A.S. da Costa } \\
\text { \& A.A. Grillo }\end{array}$ & 1 & 3 & \\
\hline Opiliaceae & M.A.S. Costa \& L.G. Lohmann & 1 & 1 & \\
\hline Oxalidaceae & $\begin{array}{l}\text { L.G. Lohmann, M.A.S. da Costa } \\
\text { \& A.A. Conceição }\end{array}$ & 1 & 1 & \\
\hline Passifloraceae & M.A.S. da Souza & 3 & 19 & 1 \\
\hline Peridiscaceae & M.J.G. Hopkins & 1 & 1 & \\
\hline Phytolaccaceae & L.G. Lohmann \& M.A.S. da Costa & 1 & 1 & \\
\hline Piperaceae & R. Callejas & 2 & 31 & \\
\hline Polygalaceae & M. do C. Marques & 4 & 15 & \\
\hline Polygonaceae & M.A.S. de Souza & 1 & 7 & \\
\hline Proteaceae & V. Plana \& K.S. Edwards & 1 & 1 & \\
\hline Quiinaceae & M. de C. Amaral & 3 & 7 & \\
\hline Rhabdodendraceae & G.T. Prance & 1 & 2 & \\
\hline Rhamnaceae & R.Lima & 1 & 1 & \\
\hline Rhizophoraceae & G.T. Prance & 2 & 2 & \\
\hline Rosaceae & M.A.S. da Costa \& L.G. Lohmann & 1 & 1 & \\
\hline Rubiaceae & M.T.V.Campos \& C.M. Taylor & 32 & 94 & 2 \\
\hline Rutaceae & J.R. Pirani & 7 & 10 & 1 \\
\hline Sabiaceae & M.A.S. da Costa & 2 & 2 & \\
\hline Sapindaceae & P. Acevedo-Rodriguez & 10 & 46 & \\
\hline Sapotaceae & T.D. Pennington & 9 & 78 & 5 \\
\hline Scrophulariaceae & $\begin{array}{l}\text { L.G. Lohmann, M.A.S. da Costa } \\
\text { \& V.C. Souza }\end{array}$ & 2 & 3 & \\
\hline Simaroubaceae & J.R. Pirani & 4 & 8 & 1 \\
\hline Siparunaceae & S.S. Renner & 1 & 9 & 1 \\
\hline Solanaceae & M. Nee & 4 & 13 & \\
\hline Sterculiaceae & J.A. da Souza \& M.F. da Silva & 3 & 7 & 1 \\
\hline Styracaceae & R. Monteiro & 1 & 2 & \\
\hline Theaceae & A. Vicentini & 1 & 2 & \\
\hline Theophrastaceae & M.A.D. de Souza & 1 & 1 & 1 \\
\hline Thymelaceae & L. Rossi & 1 & 1 & \\
\hline Tiliaceae & G. Esteves & 1 & 1 & \\
\hline Turneraceae & L.G. Lohmann \& M.A.S. da Costa & 2 & 2 & \\
\hline Ulmaceae & C.C. Berg & 2 & 2 & \\
\hline Urticaceae & C.C. Berg & 2 & 2 & \\
\hline Verbenaceae & S. Atkins & 7 & 17 & \\
\hline Violaceae & C.A. Sothers & 4 & 9 & \\
\hline Viscaceae & B. Stannard & 1 & 2 & \\
\hline Vitaceae & M.A.D. de Souza & 1 & 2 & \\
\hline \multirow[t]{2}{*}{ Vochysiaceae } & M.L. Kawasaki & 4 & 11 & \\
\hline & Total Dicotiledôneas & 505 & 1688 & 40 \\
\hline \multicolumn{5}{|c|}{ MONOCOTILEDÔNEAS } \\
\hline Araceae & M.L. Soares \& S. Mayo & 12 & 55 & 3 \\
\hline Arecaceae & A. Henderson & 13 & 43 & 2 \\
\hline Bromeliaceae & R.F. Forzza \& G. Martinelli & 9 & 15 & \\
\hline Burmanniaceae & H. Maas \& P.J.M. Maas & 6 & 9 & \\
\hline Commelinaceae & M. do C. Amaral & 2 & 3 & \\
\hline
\end{tabular}

Rodriguésia 56 (86): 9-25. 2005 


\begin{tabular}{llrrr}
\hline Familia & Especialistas & Gếneros & Táxons $^{\mathbf{2}}$ & Novas $^{\mathbf{S}^{\mathbf{2}}}$ \\
\hline Costaceae & P.J.M. Maas \& H. Maas & 1 & 3 & \\
Cyclanthaceae & F. Gomes \& R. Mello-Silva & 5 & 7 & \\
Cyperaceae & D.A. Simpson & 14 & 21 & \\
Dioscoreaceae & G. Pedralli & 1 & 7 & \\
Eriocaulaceae & A.M. Giulietti & 3 & 3 & \\
Heliconiaceae & P.J.M. Maas \& H. Maas & 1 & 2 & \\
Marantaceae & R.C. Forzza & 3 & 15 & 3 \\
Orchidaceae & J.E.L.S. Ribeiro & 41 & 75 & \\
Poaceae & F.P. Gomes \& T.S. Filgueiras & 12 & 23 & \\
Rapateaceae & R.C. Forzza \& M.A.S. da Costa & 3 & 4 & \\
Smilacaceae & R. Andreata & 1 & 5 & \\
Strelitziaceae & P.J.M. Maas \& H. Maas & 1 & 1 & \\
Thurniaceae & P.J.M. Maas \& H. Maas & 1 & 1 & \\
Triuridaceae & H. Maas \& P.J.M. Maas & 1 & 5 & \\
Xyridaceae & M. das G.L. Wanderley & 1 & 1 & \\
Zingiberaceae & P.J.M. Maas \& H. Maas & 1 & 1 & \\
& Total Monotiledôneas & $\mathbf{1 3 2}$ & $\mathbf{2 9 9}$ & $\mathbf{8}$ \\
& Total geral & $\mathbf{6 7 9}$ & $\mathbf{2 0 7 9}$ & $\mathbf{4 8}$ \\
\hline
\end{tabular}

\section{ESPÉCIES NOVAS E ENDEMISMO}

A Reserva Ducke foi selecionada para ser estudada no presente projeto principalmente por ser o local botanicamente mais bem conhecido na Amazônia brasileira. Apesar deste fato, durante a execução deste projeto aproximadamente 1.000 espécies foram adicionadas à listagem inicial que possuíamos para Reserva. Dentre essas, pelo menos 48 foram reconhecidas como espécies novas para a ciência (tabela 4). Há algumas outras espécies que ainda não foram efetivamente identificadas pelos especialistas e, provavelmente, também deverão ser tratadas como espécies novas, elevando ainda mais este número. Para algumas espécies não foram localizados materiais férteis, isto dificulta sua identificação adequada. Talvez entre essas também existam outras espécies novas.

Várias das espécies encontradas possuem seu limite de distribuição conhecido apenas para a área da Reserva Ducke. Porém é pouco provável que estas só ocorram na Reserva. Acredita-se que este resultado seja apenas o reflexo do nosso pouco conhecimento sobre as áreas mais distantes de Manaus. É arriscado afirmar que existam espécies endêmicas para a Reserva. Um exemplo disto seria é uma espécie nova de Pouteria, que será publicada por Pennington no próximo volume da flora, que também tem ocorrência na Guiana Francesa.

A coleta recente de várias espécies novas para a ciência em uma área, anteriormente a este projeto considerada bem amostrada, indica que nossos conhecimentos sobre a flora da Amazônia brasileira são ainda bastante precários. Os resultados encontrados no PFRD indicam que deveríamos investir mais em trabalhos de campo, a médio e longo prazo, na Amazônia para que possamos atingir um conhecimento adequado sobre esta enorme floresta.

\section{O futuro das floras na Amazônia}

A flora da Amazônia Central é bastante rica em termos de alfa diversidade de árvores (número de espécies por ha com DAP >10 cm) (Oliveira \& Mori, 1999) e em termos de gama diversidade (número de espécies ocorrendo regionalmente) (Prance 1990). Prance (1990) e Oliveira \& Daly (1999) apresentaram argumentos para explicar esta alta diversidade. Nelson et al. (1990) chamaram a atenção para a maior concentração de coletas próxima da cidade de Manaus e a escassez 
de dados para outras localidades da Amazônia brasileira. Como os estudos são apenas pontuais na região, consideramos que ainda não existem evidências suficientes para afirmar que a região de Manaus seja especialmente diversa comparada com outros lugares na Amazônia. Isto torna muito evidente a necessidade da realização de novos projetos semelhantes ao PFRD em outras localidades mais distantes de Manaus.

Foster (2000) e Berry (2000) destacaram a importância da contribuição original e utilidade

Tabela 5 - As trinta famílias (nesta análise separando as três subfamílias de Leguminosae) mais ricas em número de táxons (espécies, subespécies ou variedades) encontradas na Reserva Ducke.

\begin{tabular}{lc}
\hline Família & $\mathbf{n}^{\mathbf{0}}$ de espécies \\
\hline Lauraceae & 100 \\
Rubiaceae & 94 \\
Orchidaceae & 96 \\
Sapotaceae & 78 \\
Leg.: Mimosoideae & 68 \\
Leg.: Papilionoideae & 66 \\
Myrtaceae & 65 \\
Annonaceae & 60 \\
Melastomataceae & 59 \\
Araceae & 55 \\
Leg.: Caesalpinioideae & 54 \\
Bignoniaceae & 54 \\
Chrysobalanaceae & 53 \\
Clusiaceae & 49 \\
Euphorbiaceae & 48 \\
Moraceae & 47 \\
Sapindaceae & 46 \\
Arecaceae & 43 \\
Burseraceae & 42 \\
Apocynaceae & 41 \\
Lecythidaceae & 38 \\
Piperaceae & 31 \\
Myristicaceae & 25 \\
Cecropiaceae & 24 \\
Malpighiaceae & 24 \\
Meliaceae & 23 \\
Poaceae & 23 \\
Cyperaceae & 21 \\
Passifloraceae & 19 \\
Verbenaceae & 17 \\
\hline
\end{tabular}

Rodriguésia 56 (86): 9-25. 2005 do Guia de identificação das plantas vasculares da Reserva Ducke (Ribeiro et al. 1999). Rejemánek e Brewer (2001), avaliando o estado da arte da identificação de plantas tropicais usando características vegetativas, destacaram que o Guia da Ducke é absolutamente inovador neste aspecto. Esperamos que a nossa experiência neste aspecto seja aproveitada pela comunidade científica na elaboração de novos guias de identificação de plantas tropicais. Alguns outros aspectos como a importância dos auxiliares de campo, a importância do trabalho de campo a longo prazo, a alocação de pessoas dedicadas exclusivamente ao projeto, o aproveitamento do conhecimento já obtido e a participação de especialistas experientes são fundamentais para o sucesso de projetos como PFRD. Esses aspectos serão comentados mais detalhadamente a seguir.

\section{A IMPORTÂNCIA dOS AUXILIARES DE CAMPO}

$\mathrm{Na}$ região amazônica a profissão de auxiliar de campo ou mateiro é amplamente utilizada pelas empresas madeireiras, na busca e uso de produtos florestais, e em projetos de pesquisas, para coleta de material botânico. Esses profissionais também atuam na identificação de plantas para a implementação de planos de manejo e para a avaliações de impacto ambiental. Apesar de ser vastamente difundida na região, esta profissão ainda é considerada informal e os mateiros possuem pouco ou nenhum treinamento que os aproximem dos termos e conceitos da Botânica. A vivência dessas pessoas, que passam suas vidas em contato com as plantas na natureza, é fundamental e indispensável para a localização das espécies durante o trabalho de campo na região. A participação desses dedicados profissionais no PFRD facilitou enormemente a catalogação das espécies encontradas. Ressaltamos que o sucesso do Projeto da Flora da Reserva Ducke é em grande parte devido a esses dedicados profissionais. Recomenda-se que qualquer projeto de flora na região envolva a participação desses "parataxonomistas". 


\section{Dedichȩão ao Projeto}

No PFRD tivemos condições empregar vários jovens pesquisadores, e também alunos, que puderam se dedicar em tempo integral às atividades do projeto. Isso resultou em melhor desempenho de todos, pois sem os deveres administrativos e prazos que em geral envolvem pesquisadores e alunos de pósgraduação, a dedicação dos integrantes foi plena. Desta forma, recomenda-se envolver pessoas com possibilidade de dedicação exclusiva em vez de se depender apenas de pesquisadores ou alunos com outros compromissos.

\section{InVESTIMENTO EM TRABALHO DE CAMPO DE LONGO PRAZO}

As coletas que já haviam sido realizadas na Reserva antes do PFRD mostraram-se insuficientes para representar a diversidade florística da área. Se considerarmos que a Reserva era um dos locais mais conhecidos da Amazônia brasileira, a situação em qualquer outra área deve ser ainda muito pior. Considerando ainda que muitas espécies amazônicas são aparentemente bastante locais e raras, e também que muitas florescem e frutificam irregularmente ou com intervalos longos, recomenda-se então que qualquer projeto de flora tenha como prioridade um trabalho de campo intensivo e exaustivo por um longo tempo. Por causa da diversidade da Amazônia e dos problemas associados com a necessidade de obter material fértil para descrever espécies novas é essencial ter um investimento a longo prazo. Um projeto com duração inferior a cinco anos provavelmente não mostrará uma boa representatividade da flora.

\section{Programa de coletas} BASEADO EM CONHECIMENTO LOCAL

A maioria dos estudos de biodiversidade de plantas na Amazônia tem usado dados de inventários, geralmente de áreas de 1 ha e incluem apenas arbóreas. Outros estudos têm utilizado áreas maiores, e incluem plantas de menor porte. Da minha experiência no PFRD, considero que este tipo de metodologia é ineficiente para expressar composição florística local. Os principais problemas deste tipo de inventário são:

- gera poucas coletas férteis que geralmente apresentam pouca utilidade aos especialistas;

- gera muitas amostras estéreis que são difíceis de identificar e que têm pouca utilidade para ampliar o conhecimento taxonômico dos grupos;

- ignoram muitos componentes importantes da flora, como epífitas e ervas;

- são pontuais, efetivamente amostrando uma pequena porção da flora local.

Consideramos que a metodologia empregada no PFRD, ou seja, a procura de "novidades" por mateiros, alunos e especialistas, familiarizados com os grupos taxonômicos, é muito mais eficiente. Muitas vezes esta metodologia é chamada de "coleta aleatória", mas na realidade não é. Utilizando esta metodologia, as espécies mais freqüentes e conhecidas são logo inventariadas e, como tempo, o esforço de amostragem é progressivamente direcionado para as espécies mais raras, que são menos conhecidas. Outra consequiência é a redução da repetição de coleta da mesma espécie. Por exemplo, as coletas dos inventários realizados na Reserva ao longo da década de 1960 resultaram em um aumento muito pequeno no conhecimento da diversidade de espécies (fig. 2.) da Reserva Ducke. Por outro lado, com a implantação do projeto PFRD, o conhecimento da diversidade de espécies dobrou a partir das coletas realizadas na década de 1990 usando a metodologia de busca de novidades.

\section{Participação de ESPECIALISTAS EXPERIENTES}

As atividades do dia a dia do projeto, como já comentado anteriormente, são mais eficientes quando envolvem pessoas que possuem dedicação integral a ele. Por outro lado, com a 
participação de especialistas experientes no processo de coleta, identificação e elaboração dos tratamentos taxonômicos o resultado é ainda melhor. O PFRD contou com a participação de alguns dos mais renomados especialistas mundiais. O Brasil tem uma comunidade botânica grande e ativa, porém a maior parte dos pesquisadores concentrase nas Regiões Sul e Sudeste e estudam muito mais as plantas extra-amazônicas. Devido ao tamanho e à riqueza florística das áreas amazônicas, muitas localidades são subamostradas nos herbários e muitos grupos amazônicos são pouco estudados. Se tivermos em mente que temos dados concretos provando a necessidade de estudos florísticos na Amazônia e ainda noção do grande potencial que a Amazônia tem de atrair investimento (nacional e internacional), podermos tornar possível o sonho de um bom conhecimento desta flora que está sendo destruída rapidamente. Também é natural que a distribuição das espécies não respeite os limites politicamente estabelecidos para a territorialidade das nações e, assim, não é razoável esperar que a ciência botânica deva ser delineada apenas por estas fronteiras, mas sim que deva estender-se aos limites biológicos. Recomenda-se, desta forma, que projetos botânicos beneficiem-se da experiência de cientistas qualificados independentemente de sua nacionalidade ou residência.

\section{Acervo instituicional local}

O material botânico de referência para estudos futuros é da maior utilidade quando depositado em herbários locais. Um acervo que possa ser rapidamente e convenientemente consultado por pesquisadores futuros é de suma

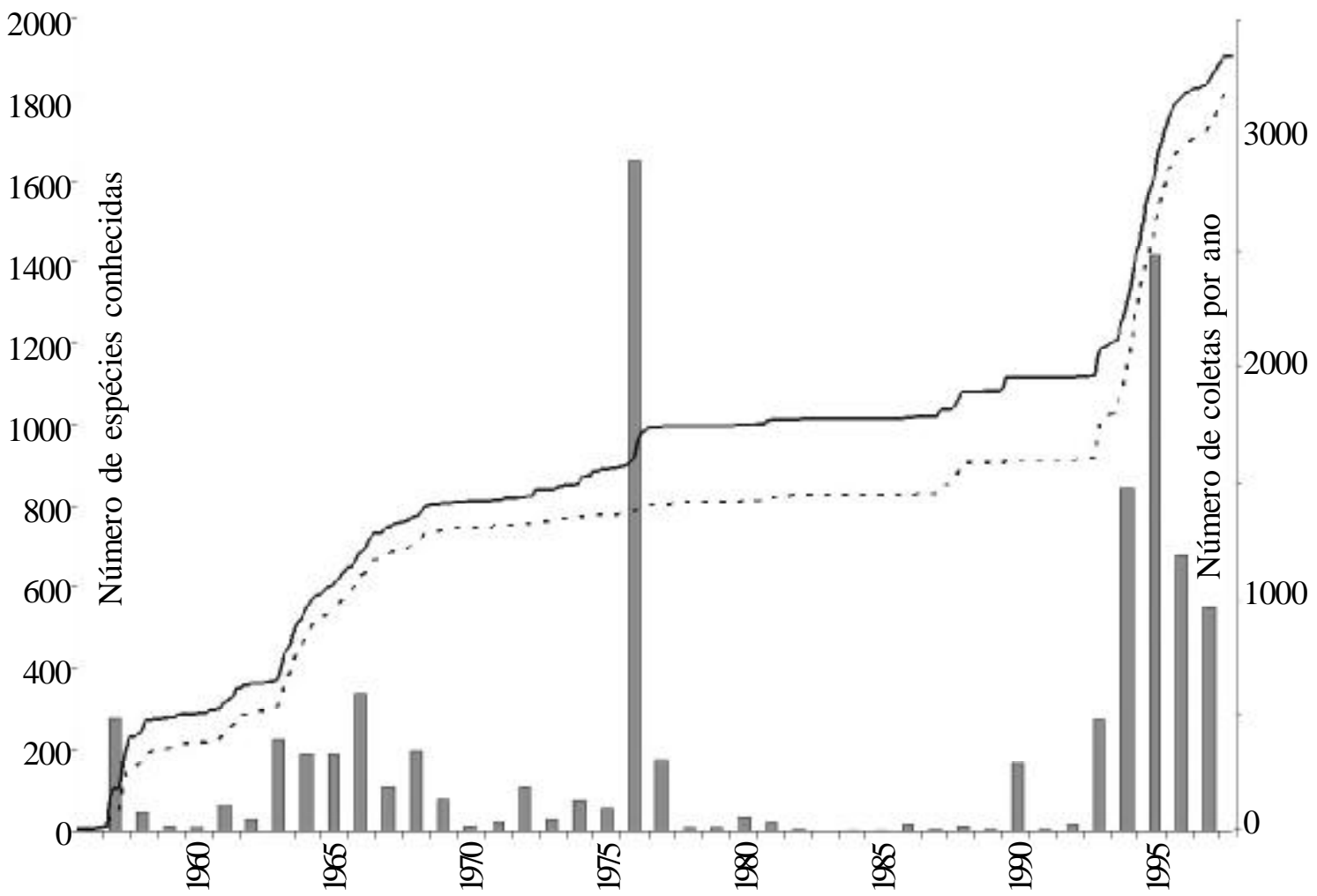

Figura 2 - Ritmo de coletas (colunas) ao longo dos anos 1954-1999 na Reserva Ducke e acúmulo de espécies conhecidas (testemunhadas por material fértil - linha pontilhada, por material estéril ou fértil - sólida). 
importância. Recomenda-se que uma representação completa do material coletado seja depositada no herbário mais perto possível do local de estudo. No caso de PFRD, isso foi o próprio herbário do INPA, com duplicatas representativas também em outros herbários amazônicos.

\section{AMPla distribuiÇão do MATERIAL}

O material coletado durante o PFRD foi distribuído para o herbário de cada especialista, e as duplicatas foram amplamente distribuídas para outros herbários, dentro e fora do Brasil. Recomendama-se que material testemunho seja coletado, sempre que possível, com muitas duplicatas (o padrão no PFRD foi de 10 duplicatas para cada espécie), e que somente sejam distribuídas após a identificação do especialista envolvido na respectiva monografia.

\section{Acervo informal para uso em pesquisa}

É melhor tentar identificar o material botânico logo depois da sua coleta. Recomenda-se que, quando possível, um acervo de referência seja mantido na unidade de pesquisa, permitindo uma rápida comparação com material coletado anteriormente. Se este acervo local puder ser mantido para consulta de pesquisadores no futuro, seria ainda melhor. Entretanto, admitese que a maioria das estações de pesquisa no Brasil não possuem condições adequadas para manutenção do acervo. Durante o projeto, um acervo, com uma duplicata de cada coleta, foi mantido em uma sala de pesquisas, perto do herbário do INPA, porém longe do local de coleta. Teria sido melhor se algum material testemunho pudesse ter sido mantido na própria sede da Reserva, mas não houve condições para isto. Então, ao fim do projeto, esta coleção de referência foi distribuída para acervos menores de pesquisa na Amazônia.

\section{LocalizaÇão da administraÇão}

Enquanto por um lado recomendo uma ampla distribuição de espécimes e o envolvimento de especialistas de todo o mundo, por outro acho muito importante que a base das operações e a administração do projeto sejam o mais próximo possível da área de estudo. No caso do PFRD, quase todas as pessoas envolvidas no dia-a-dia do projeto eram da região, ou se dispuseram a morar em Manaus durante o projeto. Isto facilitou bastante, e problemas particulares, financeiros e de logística de deslocamento foram minimizados. No caso do PFRD, a sala de pesquisa e a base da administração ficavam no INPA, a cerca de $30 \mathrm{~km}$ da área de estudo.

\section{Considerações Finais}

O conhecimento da biodiversidade verídica da Amazônia é importante não somente por razões puramente científicas e idealísticas. O bom manejo da floresta Amazônica também depende de um bom conhecimento e uma boa habilidade de separar e identificar seus componentes. Projetos como o PFRD, que aumentam o conhecimento científico e ao mesmo tempo promovem acesso a este conhecimento para pessoas da região, merecem ser apoiados.

\section{Agradecimentos}

Gostaria de agradecer a todos que ajudaram na produção do Guia e dos tratamentos taxonômicos, e ainda mais às pessoas que ajudaram através da participação na administração e em atividades cotidianas do projeto. Nomeá-las aqui geraria uma lista gigantesca. Mas agradeço profundamente a todo mundo que teve conecção com o projeto e seus produtos durante seu andamento e nos anos desde então. 
Institucionalmente, agradeço primeiro ao Instituto Nacional de Pesquisas da Amazônia, por sediar o projeto, ao Department for Overseas Development, pelo financiamento, e ao Jardim Botânico do Rio de Janeiro por viabilizar esta publicação.

Em termos de produção deste volume, agradeço ao Corpo Editorial de Rodriguésia, especialmente à Editora-chefe, Rafaela C. Forzza e à editoradora Carla M.M. Molinari, e também a Cynthia Sothers que participou comigo durante os anos de editoriação sem ter os meios de publicar.

Este artigo beneficiou-se da revisão de linguagem e conteúdo por Rafaela C. Forzza, Vidal de Freitas Mansano, Jefferson Prado e Cinthia Kameyama.

\section{Bibliografia}

Berry, P. E. 2000. Book review. Ann. Missouri Bot. Gard. 87: 433-434.

Cronquist, A. 1981. Ana integrated system of classification of flowering plants. Colombia University Press, New York. $1263 \mathrm{p}$.

Foster, R. B. 2000. Review of Flora da Reserva Ducke by Ribeiro et al. Tropinet 11(2): 1-2.

Nelson, B. W., Ferreira, C. A. C., Silva, M. F., \& Kawasaki, M. L. 1990. Endemism centres, refugia and botanical collection density in Brazilian Amazonia. Nature 345: 714-716.
Oliveira, A. A. \& Daly, D. C. 1999. Geographic distribution of tree species occurring in the region of Manaus, Brazil: implications for regional diversity and conservation. Biodiversity and Conservation 8: 1245-1259.

Oliveira, A. A.\& Mori, S. A. 1999. A central Amazonian terra firme forest. I. High tree species richness on poor soils. Biodiversity and Conservation. 8: 1245-1259.

Prance, G. T. 1990. The floristic composition of Central Amazonian Brazil. Pp. 112 140, in Gentry, A. H. Four Neotropical Forests. Yale University Press.

Rejmánek, M. \& Brewer, S. W. 2001. Vegetative Identifiation of tropical woody plants: state of the art and annotated bibliography.

Ribeiro, J. E. L. S., Nelson, B. W., Silva, M. F., Martins, L. S. S. \& Hopkins, M. J. G. 1994. Reserva Florestal Ducke: diversidade e composição da flora vascular. Acta Amazônica 24: 19-30.

Ribeiro, J. E. L. S., Hopkins, M. J. G., Vicentini, A., Sothers, C. A., Costa, M. A. S., Brito, J. M., Souza, M. A. D., Martins, L. H., Lohmann, L. G., Assunção, P. A., Pereira, E. C., Silva, C. F., Mesquita, M. R. \& Procópio, L. C. 1999. Flora da Reserva Ducke. Guia de identificação das plantas vasculares de uma floresta de terra firme na Amazônia Central. INPA-DFID, Manaus, $800 \mathrm{p}$.

Urban, I. 1906. Vitae Itineraque collectorum Botanicorum. In Martius, K. F. P. von, Fl. bras. 1(1): 1-152. 
